\title{
Adjuvant Treatment in Non-Small Cell Lung Cancer: Where Are We Now?
}

\author{
Rosalyn A. Juergens, MD, and Julie R. Brahmer, MD, MSc, Baltimore, Maryland
}

\section{Key Words}

Adjuvant, chemotherapy, lung cancer, etoposide, platinum with treatment, averages approximately 1 year. ${ }^{4}$ Second, despite definitive surgical resection of early-stage NSCLC, most patients experience relapse with local recurrence or distant metastases. ${ }^{5}$ Since the 1960s, oncologists have been investigating various adjuvant treatments to improve survival of patients with early-stage NSCLC. In the past 5 years, several published studies have changed the paradigm for treating resectable NSCLC to include adjuvant chemotherapy. ${ }^{6-10}$ This article reviews the results of these recent trials and examines the role of etoposide in combination with platinum-based chemotherapy in adjuvant treatment of NSCLC.

\section{Meta-Analysis, 1995}

In the 1960s, researchers began to assess various combinations of chemotherapy with or without radiation in adjuvant lung cancer. Many of these trials were small and therefore not suitably designed to individually assess the potential benefit of adjuvant chemotherapy in NSCLC. In 1995, the NSCLC Collaborative Group performed a meta-analysis that used individual data from 9387 patients compiled from 52 eligible randomized trials conducted between 1965 and 1991.11 This analysis was designed to investigate the usefulness of chemotherapy in lung cancer as a whole. Analyses assessed the benefits of adding chemotherapy to surgery, surgery plus radiotherapy, radiotherapy alone, and supportive care alone. The main adjuvant analysis included 14 trials (some with multiple treatment arms) that assessed the use of chemotherapy in addition to surgery: 5 early trials that examined regimens based on alkylating agents, 4 Japanese trials that assessed the use of uracil/tegafur-based regimens, and 8 trials that examined cisplatin-based adjuvant treatment. Alkylating agents were found to be harmful when used in the adjuvant setting, with a combined hazard ratio of 1.15 (95\% confidence interval [CI], 1.04-1.27). 
Juergens and Brahmer

Cisplatin-based regimens, in contrast, seemed to offer a survival advantage, with a combined hazard ratio of 0.87 (95\% CI, 0.74-1.02). This hazard ratio is consistent with a $5 \%$ improvement in 5 -year survival. Even after combining 8 trials, the size for this subanalysis was only 1394 patients. The result of this subanalysis was not statistically significant, but the trend was toward a clinically important benefit. This historic study focused investigators toward assessing cisplatin-based regimens.

\section{Recent Cisplatin-Based Trials of Adjuvant Chemotherapy}

\section{Adjuvant Lung Project Italy}

The first published modern trials assessing the usefulness of cisplatin-based adjuvant chemotherapy were designed to enroll large numbers of patients. The eligibility criteria were broad, allowing enrollment of most patients with early-stage resectable NSCLC (Table 1). One of these trials was conducted across 66 Italian centers through the Adjuvant Lung Project Italy (ALPI). ${ }^{8}$ The trial initially planned to enroll 1300 patients with stage I, II, or IIIA NSCLC. The ALPI study was designed with an $80 \%$ power to detect a $7 \%$ improvement in overall survival (57\% vs. $50 \%$ ) at 5 years. Patients randomized to the chemotherapy arm were treated with mitomycin $\mathrm{C}$, vindesine, and cisplatin (MVP) for 3 cycles. Patients on either arm of the study were also allowed to undergo adjuvant radiation according to the protocol of each participating center. More than $40 \%$ of patients on the trial underwent radiation.

After 5 years of enrollment, 1209 patients were enrolled in the study. Only $70 \%$ of patients were able to tolerate the full 3 cycles of treatment, and more than half of these patients required dose reductions or dose delays. This trial excluded 13 ineligible patients and 108 patients from a single participating center with data integrity problems, leaving 1088 evaluable patients for the main outcome of overall survival. At publication, 569 deaths had occurred across both study arms with a median follow-up of 64.5 months. The ALPI study did not show a benefit in overall survival. The hazard ratio for overall survival was 0.96 (95\% CI, 0.81-1.13). This study had a weak trend toward a benefit in overall survival, but was clearly not as promising as clinicians had hoped.

\section{International Adjuvant Lung Cancer Trial}

The International Adjuvant Lung Cancer Trial (IALT), which was conducted in 33 countries worldwide, was the second adjuvant lung cancer trial to be published. ${ }^{6}$ This study initially planned to enroll 3300 patients. This sample size would allow this study to detect a $5 \%$ improvement in overall survival at 5 years (from $50 \%$ to $55 \%$ ) with $83 \%$ power. Eligible participants included those with resected stage I, II, and III NSCLC. Because of the large number of countries

\section{Table 1 Recent Trials of Adjuvant Platinum-Based Chemotherapy in Resected NSCLC}

\begin{tabular}{|c|c|c|c|c|c|c|}
\hline Study & Patients & Stage & Regimen & $\begin{array}{l}\text { Hazard } \\
\text { Ratio }\end{array}$ & $\begin{array}{l}\text { 5-Year } \\
\text { Survival } \\
\text { (months) }\end{array}$ & $P$ value \\
\hline $\begin{array}{l}\text { Scagliotti et al. }{ }^{8} \\
\text { ALPI } 2003^{*}\end{array}$ & 1209 & I/II/IIIA & $\begin{array}{l}\text { MVdP } \\
\text { Observation }\end{array}$ & 0.96 & $\begin{array}{l}\text { NR } \\
\text { NR }\end{array}$ & .589 \\
\hline $\begin{array}{l}\text { Arriagada et al. }{ }^{6} \\
\text { IALT 2004* }\end{array}$ & 1867 & $\mathrm{I} / \mathrm{II} / \mathrm{III}$ & $\begin{array}{l}P+E, V d, V n \text {, or } V b \\
\text { Observation }\end{array}$ & 0.86 & $\begin{array}{l}44.5 \\
40\end{array}$ & $<.03$ \\
\hline $\begin{array}{l}\text { Strauss et al. }{ }^{9} \\
\text { CALGB } 2004\end{array}$ & 344 & IB & $\begin{array}{l}\text { TCp } \\
\text { Observation }\end{array}$ & 0.62 & $\begin{array}{l}71^{\dagger} \\
59^{\dagger}\end{array}$ & .028 \\
\hline $\begin{array}{l}\text { Winton et al. }{ }^{10} \\
\text { Intergroup } \\
2005\end{array}$ & 482 & IB/II & $\begin{array}{l}\text { VnP } \\
\text { Observation }\end{array}$ & 0.69 & $\begin{array}{l}69 \\
54\end{array}$ & .04 \\
\hline $\begin{array}{l}\text { Douillard et al. }{ }^{7} \\
\text { ANITA } 2005^{*}\end{array}$ & 840 & $|\mathrm{~B} /| \mathrm{I} / \mathrm{II} \mathrm{A}$ & $\begin{array}{l}\text { VnP } \\
\text { Observation }\end{array}$ & 0.79 & $\begin{array}{l}51 \\
43\end{array}$ & .013 \\
\hline
\end{tabular}

*Allowed sequential radiation therapy according to oncologist preference.

tFour-year overall survival.

Abbreviations: ALPI, Adjuvant Lung Project Italy; ANITA, Adjuvant Navelbine International Trialists Association; CALGB, Cancer and Leukemia Group B; Cp, carboplatin; E, etoposide; I, ifosfamide; IALT, International Adjuvant Lung Cancer Trialists; M, mitomycin C; NR, not reported; NSCLC, non-small cell lung cancer; P, cisplatin; T, paclitaxel; Vb, vinblastine; Vd, vindesine; Vn, vinorelbine. 
involved, this study was designed with as much flexibility as possible. Investigators were allowed to use 1 of 4 potential chemotherapy combinations, all cisplatin-based, with a total of 16 potential dose schedules. Four dosing regimens of cisplatin could be chosen: $80 \mathrm{mg} / \mathrm{m}^{2}$ for 4 cycles, $100 \mathrm{mg} / \mathrm{m}^{2}$ for 3 or 4 cycles, and $120 \mathrm{mg} / \mathrm{m}^{2}$ for 3 cycles. One of the 4 dosing schedules could be paired with vindesine, vinblastine, vinorelbine, or etoposide. More than half of the patients were treated with etoposide plus cisplatin at the $100 \mathrm{mg} / \mathrm{m}^{2}$ dose for either 3 or 4 cycles. Patients in both arms of this trial were also allowed to undergo adjuvant radiation therapy. Approximately 25\% of participants underwent radiation treatment.

After almost 5 years of accrual, the study was stopped after enrollment of 1867 patients because of a decline in the enrollment rate. Toxicity was also a limiting factor in the IALT. Only $70 \%$ of the patients randomized to the chemotherapy arm received at least 240 cumulative milligrams of cisplatin. At final analysis, the median followup was 56 months and 973 deaths combined had occurred between both arms. Twenty-five patients were excluded from the final analysis because they did not meet the eligibility criteria. The IALT did show a statistically significant survival benefit. The hazard ratio for overall survival was 0.86 (95\% CI, 0.76-0.98). This hazard ratio translates into a $4 \%$ improvement ( $44.5 \%$ vs. $40.4 \%$ ) in overall survival at 5 years. The CIs for both of these trials overlap the CI found in the 1995 meta-analysis. Many researchers have offered hypotheses about the cause of the survival difference between the ALPI and IALT. A potential increase in toxicity from the addition of a third agent to the chemotherapy regimen or from radiation therapy could explain the differences seen. The routine use of radiation in the adjuvant setting is currently not recommended. Previous studies have shown potential harm from the adjuvant use of radiation, but these studies were conducted before the widespread use of modern radiation delivery technology. ${ }^{12}$

Large trials with broad entry criteria were not the only trials conducted to assess the use of adjuvant chemotherapy in NSCLC. Two trials that were initially presented at the American Society of Clinical Oncology (ASCO) annual meeting in 2004 focused only on patients with stage I and II disease. Each trial was limited to evaluating one specific regimen.

\section{Cancer and Leukemia Group B Trial 9633}

The first of these trials was conducted through the Cancer and Leukemia Group B (CALGB). ${ }^{9}$ The
CALGB Trial 9633 targeted only patients with stage IB resected NSCLC. Patients who were randomized to the chemotherapy arm were treated with paclitaxel $200 \mathrm{mg} / \mathrm{m}^{2}$ in combination with carboplatin, dosed with an area under the curve of 6 administered every 3 weeks for 4 cycles. This trial did not allow patients to undergo adjuvant radiation.

The full results of this trial are not published, but data are available from the investigators' oral presentation at the ASCO meeting. ${ }^{9}$ The sample size calculation for the trial was not part of the presentation, but the slide presentation specified that 384 patients were initially anticipated to be accrued. This trial was stopped in 2003 by the data safety and monitoring board after accruing 344 patients, because the interim analysis documented a significant survival benefit for those patients in the chemotherapy arm.

In contrast with the previous trials, chemotherapy was very well tolerated in the CALGB trial. No deaths occurred from chemotherapy toxicity and $85 \%$ of all patients randomized to undergo chemotherapy treatment completed the planned 4 cycles. At analysis, the median follow-up was 34 months, and 88 deaths combined had occurred between both arms of the study. The chemotherapy arm showed a significant improvement in overall survival. The hazard ratio was 0.62 (95\% CI, 0.41-0.95), and an increase of 12\% in 4-year survival (71\% vs. $59 \%$ ) was observed. Updated results are anticipated from the 2006 ASCO meeting.

\section{North American Intergroup Trial JBR.10}

The North American Intergroup presented the second trial. ${ }^{10}$ Patients with resected stage IB and II NSCLC were eligible for enrollment. Patients randomized to the chemotherapy arm underwent a combination of vinorelbine and cisplatin. Vinorelbine was administered weekly for 16 weeks in combination with cisplatin $50 \mathrm{mg} / \mathrm{m}^{2}$, which was given on days 1 and 8 of each 28-day cycle for 4 cycles. This trial did not allow patients to undergo radiation therapy. The sample size for this trial was 450 patients. The trial was designed to detect a $10 \%$ improvement in 3-year survival from $60 \%$ to $70 \%$, with $80 \%$ power.

Similar to the IALT study, toxicity was a problem in the Intergroup trial. The initial dose of vinorelbine was $30 \mathrm{mg} / \mathrm{m}^{2}$ but was reduced to $25 \mathrm{mg} / \mathrm{m}^{2}$ after the first 18 patients were enrolled because of unacceptable toxicity. Two treatment-related deaths occurred. Only $45 \%$ of patients completed the full 4 cycles of prescribed chemotherapy. Hematologic 
toxicity was most prominent, and $15 \%$ of patients on treatment required colony-stimulating factors. At analysis, 482 patients had been randomized and followed up for a median of 5.2 years. Between the 2 arms of the study, 197 deaths had occurred. The Intergroup trial, despite significant toxicity, showed a significant survival advantage. The hazard ratio in this trial was 0.69 (95\% CI, 0.52-0.91). A 15\% improvement in 5-year survival was observed in patients who underwent adjuvant chemotherapy.

\section{Adjuvant Navelbine International Trialist Association}

The most recent trial, presented at the 11th World Conference on Lung Cancer, was conducted by the Adjuvant Navelbine International Trialist Association (ANITA). ${ }^{7}$ Patients were enrolled in 14 countries. This design returns to the paradigm of the earlier trials, which had broad entry criteria with the goal of accruing a large number of patients. Patients with stage IB through IIIA NSCLC who underwent complete resection were eligible for enrollment. The chemotherapy regimen for the ANITA trial specified vinorelbine $30 \mathrm{mg} / \mathrm{m}^{2}$ weekly for 16 weeks in combination with cisplatin $100 \mathrm{mg} / \mathrm{m}^{2}$ on day 1 of each 28 day cycle for a planned 4 cycles. Radiation therapy was allowed at the discretion of the treating center. The planned sample size for this trial was 800 patients. The trial was designed to detect a 10\% improvement in 2-year survival with $90 \%$ power.

Toxicity was again a problem in this trial. Five treatment-related deaths occurred. Slightly more than half of the planned vinorelbine and three quarters of the cisplatin could be administered. At analysis the trial had enrolled 840 patients who had been followed up for a median of 70 months. The data from this trial are only available in abstract form. At presentation, 452 patients had died between the 2 arms. The ANITA trial showed a survival advantage consistent with the previous vinorelbine and cisplatin-based trials. The hazard ratio for overall survival was 0.79 (95\% CI, 0.66-0.95). This hazard ratio translates into an improvement of $8.6 \%$ in 5 -year survival for those patients treated with chemotherapy.

\section{Recent UFT-Based Trials of Adjuvant Chemotherapy}

All of the trials discussed previously involved intravenously administered chemotherapy. Several Japanese trials have evaluated the use of oral chemotherapy in the adjuvant setting. In 2004, a large study by Kato et al. ${ }^{13}$ randomized 979 patients with stage I disease to undergo either observation or 2 years of UFT therapy. Both groups showed excellent overall survival of $85 \%$ and $88 \%$, respectively, and the difference reached statistical significance $(P=.047)$.

A meta-analysis of data on 2003 patients from 6 trials assessing adjuvant UFT use was presented at the 2004 ASCO meeting. ${ }^{14}$ The hazard ratio for those treated with UFT was 0.77 (95\% CI, 0.63-0.94) compared with observation alone. This translated into an overall 5 -year survival benefit of 5.3 years, which was confirmed in a subgroup analysis of another metaanalysis by Hotta et al. ${ }^{15}$ Single-agent adjuvant UFT therapy was shown to have a beneficial hazard ratio of 0.799 (95\% CI, 0.668-0.957). Currently, all data on this agent have been collected outside the United States.

\section{Meta-Analysis, 2005}

Since the 1995 meta-analysis, numerous trials have added to the data collected between 1965 and 1991. Several groups have published and presented updated analyses based on the current data. ${ }^{15-17}$ One of these analyses focused specifically on cisplatin-based randomized trials and included the 2 trials presented at the ASCO meeting in $2004 .{ }^{16}$ Eighteen trials were compiled for this analysis, which included data from 6494 patients. The updated overall hazard ratio was 0.93 (95\% CI, 0.89-0.95). This translates into an overall survival advantage of $6 \%$ to $13 \%$ at 5 years. Subgroup analyses were also performed. The hazard ratio for patients with stage I and II disease in the trials was 0.87 (95\% CI, 0.80-0.95). The hazard ratio for patients with stage III disease in the trials was 0.93 (95\% CI, 0.87-1.00). This result was not statistically significant, but this subanalysis only included 1341 patients.

\section{Role of Etoposide With Platinum}

Etoposide combined with platinum chemotherapy is the mainstay of treatment for small cell lung cancer (SCLC). This regimen also has significant activity in NSCLC. Use of etoposide and platinum is expanding, especially in the setting of locally advanced NSCLC. The use of etoposide and platinum stems 
from the advantages of the regimen that are exploited in the treatment of limited stage SCLC. The standard etoposide and platinum regimen can be combined at full dose with radiation therapy. Patients are deriving benefit through increased radiation sensitization from the chemotherapy and from undergoing full systemic doses designed to maximally reduce the incidence of metastatic recurrence. A trial conducted by the Southwest Oncology Group demonstrated this concept in NSCLC..$^{18}$ This trial assessed the use of concurrent etoposide, cisplatin, and radiation followed by docetaxel consolidation for stage IIIB NSCLC. The median survival of patients in this trial was 26 months. This is almost double the median survival of previously conducted trials in this patient population, although it is a phase II trial.

The potential advantages of using cisplatin and etoposide in the adjuvant setting have been assessed. The first trial published was conducted through the Eastern Cooperative Oncology Group in 2000. ${ }^{19}$ The schema for this trial compared the adjuvant use of concurrent cisplatin, etoposide, and radiation to radiation alone. This trial enrolled 488 patients with completely resected stage II and III NSCLC. No difference was seen between the group who underwent combined modality treatment and the group treated with radiation alone. Based on the experience of other adjuvant trials, the sample size of this trial is inadequate to determine the usefulness of combined chemotherapy and radiation compared with radiation alone. The 39\% overall 5-year survival in this study was remarkable given the stage of the patients. The results of this trial are difficult to interpret based on current knowledge. To appropriately assess the usefulness of combined chemotherapy and radiation therapy in the adjuvant setting, a trial comparing both modalities with adjuvant chemotherapy alone would need to be conducted.

Cisplatin and etoposide have also been used without radiation therapy in adjuvant NSCLC regimens. A trial conducted in Italy assessed the use of cisplatin and etoposide as postoperative adjuvant therapy for stage IB NSCLC. ${ }^{20}$ The study was small, with a planned sample size of only 66 patients. A statistically significant $18 \% 5$-year survival advantage was shown for the chemotherapy group. Given the small sample size, this study is intriguing but not conclusive. The data from this trial, however, are confirmed through the IALT study. More than 1000 patients in the IALT study underwent treatment with cisplatin and etoposide. This trial showed the first published survival advantage from adjuvant chemotherapy across all stages of resectable NSCLC. Multiple platinum-based regimens have been studied for use in the adjuvant setting in NSCLC. Cisplatin and etoposide is one potential choice, but no single best regimen has been found.

\section{Conclusions}

Lung cancer is a deadly disease in the United States and worldwide. Millions of patients could benefit from improved efficacy and tolerability of adjuvant chemotherapy in lung cancer. In the United States alone, a 5\% improvement in 5-year survival could translate into more than 3000 lives saved annually. Based on the data collected, the National Comprehensive Cancer Network has included adjuvant chemotherapy for lung cancer as a category 1 recommendation for stages IB through III resected NSCLC. Future studies must be conducted to further assess the optimal regimen for safety and efficacy of adjuvant NSCLC. With numerous potential choices, regimen tolerability becomes a key factor. Well-tolerated older regimens, such as etoposide and cisplatin, are used widely in the adjuvant setting in Europe and with increased frequency in the United States. Currently, treating physicians are challenged with tailoring the treatment regimen to the patient. The challenge will be to select those patients who will experience the most benefit and the least risk.

\section{References}

1. Jemal A, Murray T, Ward E, et al. Cancer statistics, 2005. CA Cancer J Clin 2005;55:10-30.

2. American Cancer Society. Estimated new cancer cases and deaths by sex for all sites, US, 2006. Available at: www.cancer.org. Accessed February 14, 2006.

3. Schiller JH, Harrington D, Belani CP, et al. Comparison of four chemotherapy regimens for advanced non-small-cell lung cancer. N Engl J Med 2002;346:92-98.

4. Sandler AB, Gray R, Brahmer J, et al. Randomized phase II/III trial of paclitaxel (P) plus carboplatin (C) with or without bevacizumab (NSC \#704865) in patients with advanced non-squamous non-small cell lung cancer (NSCLC): an Eastern Cooperative Oncology Group (ECOG) Trial_E4599 (abstract). J Clin Oncol 2005;23(16 suppl):LBA4.

5. Immerman SC, Vanecko RM, Fry WA, et al. Site of recurrence in patients with stages I and II carcinoma of the lung resected for cure. Ann Thorac Surg 1981;32:23-27.

6. Arriagada R, Bergman B, Dunant A, et al. Cisplatin-based adjuvant chemotherapy in patients with completely resected non-small-cell lung cancer. N Engl J Med 2004;350:351-360.

7. Douillard JY, Rosell R, Delena M, et al. ANITA: phase III adjuvant vinorelbine $(\mathrm{N})$ and cisplatin $(\mathrm{P})$ versus observation (OBS) in completely resected (stage I-III) non-small-cell lung cancer (NSCLC) 
Juergens and Brahmer

patients (pts): final results after 70-month median follow-up. On behalf of the Adjuvant Navelbine International Trialist Association (abstract). J Clin Oncol 2005;23(16 suppl):7013.

8. Scagliotti GV, Fossati R, Torri $V$, et al. Randomized study of adjuvant chemotherapy for completely resected stage I, II, or IIIA nonsmall-cell lung cancer. J Natl Cancer Inst 2003;95:1453-1461.

9. Strauss $G$, Herndon J, Maddaus $M$, et al. Randomized clinical trial of adjuvant chemotherapy with paclitaxel and carboplatin following resection in stage IB non-small cell lung cancer (NSCLC): report of Cancer and Leukemia Group B (CALBG) Protocol 9633. J Clin Oncol 2004;22(14 suppl):7019.

10. Winton $T$, Livingston $R$, Johnson $D$, et al. Vinorelbine plus cisplatin vs. observation in resected non-small-cell lung cancer. N Engl J Med 2005;352(25):2589-2597.

11. Chemotherapy in non-small cell lung cancer: a meta-analysis using updated data on individual patients from 52 randomised clinical trials. Non-small Cell Lung Cancer Collaborative Group. BMJ 1995;311:899-909.

12. Postoperative radiotherapy in non-small-cell lung cancer: systemic review and meta-analysis of individual patient data from nine randomised controlled trials. PORT Meta-analysis Trialists Group. Lancet 1998;352:257-263.

13. Kato $\mathrm{H}$, Ichinose $\mathrm{Y}$, Ohta $\mathrm{M}$, et al. A randomized trial of adjuvant chemotherapy with uracil-tegafur for adenocarcinoma of the lung. N Engl J Med 2004;350:1713-1721.
14. Hamada C, Ohta M, Wada $H$, et al. Survival benefit of oral UFT for adjuvant chemotherapy after completely resected non-small-cell lung cancer. J Clin Oncol 2004;22:7002.

15. Hotta $\mathrm{K}$, Matsuo $\mathrm{K}$, Ueoka $\mathrm{H}$, et al. Role of adjuvant chemotherapy in patients with resected non-small-cell lung cancer: reappraisal with a meta-analysis of randomized controlled trials. J Clin Oncol 2004:22:3860-3867.

16. Bria E, Gralla RJ, Raftopoulos $\mathrm{H}$, et al. Does adjuvant chemotherapy improve survival in non small cell lung cancer (NSCLC)? A pooled-analysis of 6494 patients in 12 studies, examining survival and magnitude of benefit (abstract). J Clin Oncol 2005;23(16 suppl):7140.

17. Sedrakyan A, Van Der MJ, O'Byrne K, et al. Postoperative chemotherapy for non-small cell lung cancer: A systematic review and meta-analysis. J Thorac Cardiovasc Surg 2004;128:414-419.

18. Gandara DR, Chansky K, Albain KS, et al. Consolidation docetaxel after concurrent chemoradiotherapy in stage IIIB non-small-cell lung cancer: phase II Southwest Oncology Group Study S9504. J Clin Oncol 2003;21:2004-2010.

19. Keller SM, Adak $\mathrm{S}$, Wagner $\mathrm{H}$, et al. A randomized trial of postoperative adjuvant therapy in patients with completely resected stage II or IIIA non-small-cell lung cancer. Eastern Cooperative Oncology Group. N Engl J Med 2000;343:1217-1222.

20. Mineo TC, Ambrogi V, Corsaro V, Roselli M. Postoperative adjuvant therapy for stage IB non-small-cell lung cancer. Eur J Cardiothorac Surg 2001;20:378-384. 